Revue d'histoire de l'Amérique française

BRS REVUE D.HISTOIRE DE L'AMÉRIQUE FRANÇAISE

\title{
Conception de la littérature chez Joseph-Sabin Raymond
}

\section{Guy Provost}

Volume 32, numéro 4, mars 1979

URI : https://id.erudit.org/iderudit/303729ar

DOI : https://doi.org/10.7202/303729ar

Aller au sommaire du numéro

Éditeur(s)

Institut d'histoire de l'Amérique française

ISSN

0035-2357 (imprimé)

1492-1383 (numérique)

Découvrir la revue

Citer cet article

Provost, G. (1979). Conception de la littérature chez Joseph-Sabin Raymond. Revue d'histoire de l'Amérique française, 32(4), 585-602.

https://doi.org/10.7202/303729ar d'utilisation que vous pouvez consulter en ligne.

https://apropos.erudit.org/fr/usagers/politique-dutilisation/ 


\title{
CONCEPTION DE LA LITTÉRATURE CHEZ JOSEPH-SABIN RAYMOND*
}

\author{
GUY PROVOST \\ Département de français \\ Collège militaire royal \\ Saint-Jean, Qué.
}

Tous les historiens de la littérature du Québec reconnaissent la part considérable accordée à la religion et à la morale par la critique littéraire au $\mathrm{XIX}^{\mathrm{e}}$ siècle. Ils rappellent aussi, à l'occasion, les litanies des écrivains, des critiques littéraires à la petite semaine, des journalistes qui, pieux défenseurs de la foi et des bonnes mœurs, dogmatisaient sur le fait littéraire d'ici ou d'ailleurs. Sur cet état de fait, et avec raison, les analystes font surtout valoir des explications d'ordre social ou religieux. Toutefois, la compréhension des réserves de la critique souffre du manque de textes du XIX ${ }^{\mathrm{e}}$ siècle qui élaborent une rationalisation de l'usage de la morale pour fin de critique littéraire.

En premier lieu, cet article tentera de combler ce vide en présentant deux textes dans lesquels l'abbé Joseph-Sabin Raymond ' ${ }^{1}$, éducateur et directeur du Séminaire de Saint-Hyacinthe pendant plusieurs années, tire l'argumentation qui fonde sa vision de la littérature.

* Il vous semblera peut-être curieux qu'un sujet comme celui-là soit offert à une revue d'histoire. Pourtant c'est sans même considérer l'offrir à une revue littéraire que je vous l'adresse, car je crois qu'il intéresse surtout les historiens, ceux de l'évolution des mentalités, des idéologies, de la pédagogie et... de la littérature. D'ailleurs, seuls des historiens se sont vraiment interrogés sur ce «personnage » jusqu'à aujourd'hui (note de l'auteur).

1 Né le 13 mars 1810 à Saint-Hyacinthe, Joseph-Sabin Raymond, décède le 3 juillet 1887. Il a été ordonné prêtre en septembre 1832 et nommé grand-vicaire en décembre 1852. Au Séminaire de Saint-Hyacinthe, il fut professeur de 1832 à 1847, de 1853 à 1859 , de 1865 à 1877 et de 1883 à 1887 . Il dirigea la maison de 1847 à 1853 , de 1859 à 1865 et de 1877 à 1883. (J.-B.-A. Allaire, Dictionnaire biographique du clergé canadien-français (Montréal, École catholique des sourds-muets, 1910), 465. 
Sauf erreur, ce curieux homme ${ }^{2}$, dont on n'a pas encore déterminé toute l'influence, est le premier à avoir consigné non pas une théorie de la littérature, mais une façon de considérer le fait littéraire, à l'aide de la théorie du sens commun de Lamennais, qui s'accorde avec une conception religieuse de la vie. Car, pour ce Mascoutain, vision du monde et vision de la littérature se répondent et s'harmonisent. Et puisque le champ littéraire au $\mathrm{XIX}^{\mathrm{e}}$ siècle est beaucoup plus vaste qu'aujourd 'hui ${ }^{3}$, c'est souvent au hasard des apartés paralittéraires de Raymond que nous pouvons les saisir. Aussi avons-nous cru profitable pour le lecteur d'établir, en deuxième lieu, l'inventaire des idées jusqu'à aujourd'hui parcellées de Raymond sur la littérature et ses objets connexes.

Des écrits de Raymond que nous avons consultés (sermons, conférences, discours de circonstance, entretiens lus aux cérémonies de fin d'année, querelles journalistiques) se dégagent deux constantes: une volonté de publier les textes au moment opportun et un souci d'ordre didactique ${ }^{4}$. En littérature, par exemple, dans les années 60, certaines des opinions de l'abbé paraissent alors que se dessine une mise en garde contre la France littéraire athée et qu'est soulevée la question du gaumisme ${ }^{5}$, question pédagogique réglée depuis près de dix ans au Séminaire de Saint-Hyacinthe. Ces préoccupations, marquées de l'enthousiasme de l'ancien professeur de littérature, d'éloquence, d'histoire et de philosophie, trouvent surtout leurs origines dans le caractère sacerdotal de l'auteur. Elles proviennent aussi de son rôle de supérieur et d'homme d'influence, pour qui l'écrit permet

2 "Âme agissante du Séminaire" selon l'historien de la maison, Raymond est aussi un contemplatif. Ultramontain de l'école de $\mathrm{Mgr}$ Bourget et gaumiste avant l'heure (voir p. 2 n. 5), il se voit classer dans le camp des catholiques libéraux en 1872. En politique, ses sympathies vont au parti conservateur.

3 Il embrasse des domaines qui relèvent de la science ou qui lui sont maintenant étrangers: l'économie, le droit, l'histoire, la philosophie, l'apologétique.

4 Ce besoin d'enseigner, de convaincre est poussé à l'extrême dans certains textes. Les procédés tiennent plus des techniques de la propagande que de ceux de l'éloquence. Voir, par exemple, les discours: «Devoirs envers le pape» (1861) et «Discours sur la nécessité de la force morale» (1865).

5 «Une manifestation de l'école ultramontaine» traitant d'une «question de pédagogie à incidences religieuses et politiques" et dont la thèse fut défendue par Mgr Gaume en 1851. Selon lui, les éducateurs initiaient trop les jeunes aux belleslettres et à la société païenne au lieu de présenter des modèles d'inspiration chrétienne tirés de la Bible et des écrits des Pères de l’Église. L'abbé Raymond se préoccupe de cette question dès les années 30. Au Séminaire de Saint-Hyacinthe, les étudiants lisaient les Pères de l'Église avec les auteurs païens en 1853. (Robert Sylvain, "Gaumisme en vase clos», Rev'ue de l'Université Lav'al, IV, 3 (1949); 254. 
de défendre le collège qu'il dirige ou de faire valoir ses opinions sur les questions de l'heure. D'ailleurs, l'abbé Raymond suit de près l'actualité au Canada comme en France.

Les travaux de Claude Galarneau et de Robert Sylvain ont montré l'engouement que manifestait l'abbé Raymond à l'égard de ses maîtres à penser d'outre-mer ${ }^{6}$. On sait qu'un professeur de rhétorique initia Raymond, encore adolescent, à l'auteur du Génie du Christianisme, dont les ouvrages, plus tard, seront «lus et relus sans cesse ${ }^{7}$; que jeune prêtre, Raymond s'enthousiasma en romantique ardent qu'il était alors, ou en contemplatif qu'il fut toujours, pour les grands catholiques du renouveau religieux en France, surtout Lamennais et ses disciples Montalembert et Lacordaire. Il les lit, correspond avec eux ou les rencontre lors de ses voyages en Europe en 1842-438 . L'historien Galarneau conclut avec justesse que la correspondance de Raymond «...affirme son désir de trouver une apologétique moderne du catholicisme ${ }^{9}$. Nous renchérissons sur cette conclusion en disant que la plupart de ses écrits portent la marque de cette orientation. De plus, quand Raymond se mêle de citer ses auteurs de prédilection, il choisit de préférence les arguments que commandent le ravissement, l'émerveillement devant le majestueux, le grandiose, le sublime religieux: vision que, surtout ses deux premiers maîtres à penser, Chateaubriand et Lamennais ont en commun. Son admiration pour l'auteur de René, il la dit toute sa vie, tandis que l'enthousiasme qu'il manifeste à l'égard de l'auteur de l'Essai sur l'indifférence se transforme en sympathie par nécessité après la défection du solitaire de La Chenaie ${ }^{10}$. La philosophie mennaisienne laissa néanmoins des traces, surtout les idées «du Lamennais de la première manière [...] dont la doctrine, le traditionalisme, tendait à une revendication absolue des droits de la religion, parce qu'elle faisait du surnaturel la condition même de toute vie intellectuelle ${ }^{11}$, celle qui détermina le

\footnotetext{
6 Claude Galarneau, «L'Abbé Joseph-Sabin Raymond et les grands romantiques français (1834-1857)», CHAR (1963): 81-88. Robert Sylvain, "Le premier disciple de Montalembert: l'abbé Joseph-Sabin Raymond», RHAF, XVI, 1 (1963): [93]-103. 85.

7 Raymond à Chateaubriand, 4 avril 1834 , cité par Claude Galarneau, op. cit. :

8 Il correspond avec Chateaubriand, Lamennais, Montalembert, Lacordaire, Gerbet, Guéranger; il les rencontre tous sauf Lamennais. Il a aussi des entretiens avec, entre autres, Mgr de Forbin-Janson, Laurentie, Ozanam (Ibid. : passim.)

9 Ibid.: 88.

10 Ibid.: 82ss.

11 Robert Sylvain, «Libéralisme et ultramontanisme au Canada français: affrontement idéologique et doctrinal (1840-1865)", Le Bouclier d'Achille. Regards sur le Canada de l'ère victorienne (Toronto, McClelland and Stewart, 1968), 1: 134.
} 
courant ultramontain. C'est par la théorie du sens commun que JosephSabin Raymond va faire la quête de la spécificité universelle de la littérature; c'est en elle qu'il va puiser et élaborer une dialectique qui pourra servir de base à ses opinions ultérieures.

L'application des idées fondamentales de Lamennais à la littérature n'est pas entièrement nouvelle. Comme le souligne Louis Le Guillou $^{12}$, elle a fait l'objet des commentaires de certains de ses disciples, Gerbet et Salinis entre autres, et ressemble à la théorie, chère aux classiques, selon laquelle la valeur d'une œuvre repose sur la constante admiration de la postérité ${ }^{13}$. Les mennaisiens allaient toutefois plus loin. Ils incorporaient l'admiration soutenue à travers les âges à l'égard d'une œuvre, une théorie du beau, à «la raison générale [qui] n'est que la raison de Dieu même ${ }^{14}$, une théorie du vrai: cette "raison générale ou l'autorité» se constituant de «l'uniformité des perceptions et [de] l'accord des jugements ${ }^{15}$.

Dès 1824 , Salinis voyait du dérèglement dans le principe d'individualité du romantisme et du consentement général dans l'universalité de l'école classique. «De là ces dogmes littéraires (ceux du classicisme), disait-il, expression du goût de l'homme, en tant qu'il est le même dans tous les temps, chez tous les peuples, dans tous les pays, emprunteront de ce consentement général une autorité sacrée et à laquelle on ne peut toucher sans rien ébranler. ${ }^{16}$ " Le "Point de littérature, si on n'en cherche la base dans le goût général ${ }^{17} \mathrm{de}$ Gerbet vient lui aussi de l'idée que la question littéraire se résout avec les principes qui solutionnent la question de la certitude.

\footnotetext{
12 Louis Le Guillou, L'Évolution de la pensée religieuse de Félicité Lamennais (Paris, Armand Colin, 1966), 90-92.

13 Boileau dans Préface: "Le gros des Hommes peut bien, durant quelques temps, prendre le faux pour le vrai, et admirer de méchantes choses: mais il n'est pas possible qu'à la longue une bonne chose ne luy plaise». [Boileau, Oeuvres complètes (Coll. Pléiade, 1966), 3]. Il écrit dans «Réflexion VII» sur Longin: «Il n'y a en effet que l'approbation de la Postérité, qui puisse établir le vrai mérite des Ouvrages [...] Le Gros des Hommes à la longue ne se trompe point sur les ouvrages d'esprit $[\ldots]$ il s'agit de sçavoir en quoy consiste ce merveilleux qui les (Homère, Platon, Ciceron, Virgile) a fait admirer de tant de siècles; et il faut trouver moyen de le voir, ou renoncer aux belles lettres ausquelles vous devez croire que vous n'avez ni goust ni génie, puisque vous ne sentez point ce qu'ont senti tous les hommes.» (Ibid., $523 \mathrm{ss}$.)

14 Cité par Le Guillou, op. cit., 203.

15 Ibid.

16 Le Mémorial catholique (janvier 1824), cité par Le Guillou, op. cit., 92.

17 Cité par Le Guillou, op. cit., 91.
} 
C'est cette base, marquée du sceau de l'aveu universel, valable pour toute la connaissance humaine, qui commande foi, autorité et obéissance à des devoirs communs, plus que le libre exercice de la volonté individuelle, que retiendra surtout le néophyte canadien.

Deux documents inédits ${ }^{18}$, non datés, l'un ${ }^{19}$ présenté sous forme d'entretien, - forme qu'affectionne l'abbé pour les cérémonies publiques, - l'autre ${ }^{20}$, paraphrase et copie du premier et plus explicite quant à la littérature, exposent les idées de Raymond.

Comme tant d'autres se limiteront à la ressasser après lui ${ }^{21}$, le jeune professeur emprunte une phrase à Platon: «Le Beau est la splendeur du vrai», et veut en démontrer la véracité à l'aide de la théorie du sens commun, qu'il défend, à l'occasion, contre les « sophismes cartésiens» de ses détracteurs ${ }^{22}$.

Pour Raymond, il existe chez tous les peuples «une révélation primitive» ${ }^{23}$, "source originelle de toutes les pensées vraies et de tous les beaux sentiments ${ }^{24}$, un consensus sur l'essence du beau, un fond de «quelque chose de commun et d'universel» qui apparente la beauté à la vérité:

...le beau n'est autre chose que le vrai, il n'est pas surprenant que la vraie beauté se reconnaisse à la même marque que la vérité. Tout se lie dans l'ordre intellectuel: tout doit se ramener à un même principe commun aux diverses classifications de cette ordre. L'unité est la loi générale établie par le souverain être: une doctrine qui présente ce caractère, et se trouve applicable aux notions du beau comme du vrai, porte par là même une démonstration de la vérité. 25

18 Les documents remontent probablement à la période $1830-1834$, puisque la ferveur mennaisienne de Raymond débute en 1830. [C.-P. Choquette, Histoire du Séminaire de Saint-Hyacinthe depuis sa fondation jusqu'à nos jours (Montréal, Imprimerie de l'Institut de Sourds-Muets, 1911-12), 1: $128 \mathrm{~s}$.]

19 [Joseph-Sabin Raymond], «Entretien sur l'éloquence et la littérature», ASSH, Fonds Raymond (F. R.), FG-3, A-5, R. 2, dos. 11, 65 p.

F. R. ibid., 13 p.

[Raymond], "Quelques réflexions sur la littérature et les arts», ASSH,

21 Cf. Yves Dostaler. Les Infortunes du roman dans le Québec du XIX e siècle (Montréal, Hurtubise HMH, 1977), 52 ss.

22 [Raymond], Entretien..., 14, 15.

23 Ibid., 9.

24 [Raymond], Quelques réflexions..., 4.

25 [Raymond], Entretien..., 3s. 
Et «...comme la marque distincte de la vérité est le consentement commun; ainsi le beau est ce qui est beau universellement ou du moins généralement ${ }^{26}$.

Cette certitude, qui commande un acte de foi, fait table rase des goûts contraires ou particuliers. Elle ne protège nullement contre l'erreur humaine. Le philosophe suggère alors deux façons d'éprouver «l'inspiration» dans les cas litigieux: l'une «consiste à comparer ce que nous avons cru voir, aux vérités traditionnelles et communes» ${ }^{27}$; l'autre exige de s'en reporter, après consultation, au «jugement général des hommes compétents sur cette matière ${ }^{28}$. L'une et l'autre de ces méthodes permettent de rétablir la preuve «que le goût général est la règle qui doit diriger les jugements particuliers $"{ }^{29}$.

Défendre le beau, littéraire ou autre, à partir de la théorie du sens commun, appuyé des arguments qui servent à la démonstration de la certitude, c'est courir le risque, surtout pour un religieux, de lier le beau à la morale générale et, finalement, aux doctrines du catholicisme. Ce pas, Raymond le franchit allégrement, car, partout, dans tous les temps, "en dernière analyse", l'homme recherche l'infini. À preuve, soutient-il, l'admiration unanime soulevée par certains passages dans les œuvres qui tentent d'éveiller «le sentiment des réalités éternelles ", tant chez les anciens que chez les modernes ${ }^{30}$ :

La littérature ne peut plaire qu'autant qu'elle réveille dans nos âmes le sentiment de l'infini, et c'est à l'autorité générale qu'il appartient de faire connaître les vrais rapports qui existent entre l'infini et les sujets bornés sur lequel le génie s'exerce. ${ }^{31}$

Par conséquent, les beaux arts ou la littérature partagent le même objet que la religion, ce qui, d'ailleurs, amène Raymond à qualifier la littérature de «fille et compagne inséparable de la religion» ${ }^{32}$. Dans cette parenté, il est évident qu'il y a subordination:

Au fond la morale de la religion n'est pas autre que celle du sang [sens] commun et tout ce qu'elle demande à la littérature, c'est

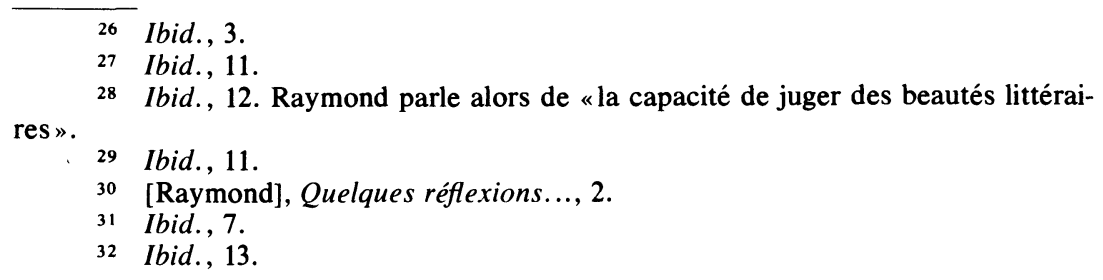


de respecter constamment ces lois sacrées auxquelles l'univers entier rend hommage. ${ }^{33}$

Et puisque le catholicisme repose sur ce fond de «lois sacrées», la conclusion se tire d'elle-même:

Donc, ou la littérature peut vivre et faire les délices du monde, et ravir par mille chefs-d'œuvre l'admiration des peuples et des âges en se soumettant sans réserve aux lois de l'église catholique, ou il faut dire que la morale universelle est un obstacle à son succès. ${ }^{34}$

Appuyé sur de tels fondements philosophiques ${ }^{35}$, Raymond va baliser les voies à suivre, évaluer les œuvres à la grille du beau moral et assigner des rôles et des objectifs à la littérature et à ses adeptes, écrivains et lecteurs.

Il charge «l'homme de génie» d'une mission qui n'est rien de moins que de rendre les hommes «meilleurs et plus heureux». Et, de la littérature, il exige qu'elle joue de son influence «au plus haut degré possible» pour perfectionner les «mœurs publiques et privées». Bien entendu, ces buts ne seront atteints qu'avec des moyens «fondé[s] sur le christianisme ${ }^{36}$.

Cette subordination de l'écrivain et de la littérature à un objet autre que le sien présage d'un utilitarisme qui mène droit à l'apologétique et au prosélytisme. D'ailleurs, la conclusion du document, qui définit ce que devrait être la «littérature catholique», en fait foi:

33 [Raymond], Entretien..., 21. Le mot religion est remplacé par le mot Église dans le deuxième document (Quelques réflexions..., 9).

34 Ibid., 21.

35 De l'étude d'une polémique sur le sens commun avec l'abbé Odelin, Louise Marcil-Lacoste conclut: "Ce qui est intéressant ici, c'est que Raymond propose une théorie de la certitude qui est au fond une théorie de l'erreur. Apparemment, laissé à lui-même, l'homme est fondamentalement erratique, d'où d'ailleurs la nécessité d'un enseignement, qui, par le biais du sens commun, donnera une norme de rectification des jugements individuels." [Louise Marcil-Lacoste, "Sens commun et philosophie québécoise: trois exemples», dans Philosophie au Québec (Montréal, Bellarmin, 1976), 84.] Hypothèse: la conception de l'enseignement de la littérature irait dans le même sens pour Raymond. Le texte littéraire sert de modèle au mieux dire et doit mener au mieux penser. Conséquemment, Raymond préviendrait les errements de la pensée et du goût par l'enseignement. "L'homme est un être nécessairement enseigné », retient-il. [J.-S. Raymond, Dissertation sur le pape (Montréal, Eusèbe Sénécal, 1870), 17.]

36 [Raymond], Quelques réflexions..., 1. 
La littérature catholique, telle qu'elle devrait être consisterait à développer avec détail les éléments du Beau que le christianisme renferme, à montrer la fécondité inépuisable de nos dogmes; tous les rapports infinis, toutes les harmonies ravissantes, qu'ils ont avec notre nature, et à manifester au monde tout ce qu'il y a de sentiments sublimes au fond du cœur des vrais chrétiens, tout ce que renferme de merveilles ces cœurs où Dieu lui-même habite, et qui sont l'image du ciel.

\section{(...)}

Toujours d'accord avec la plus pure morale, toujours fidèle, lorsqu'elle peindrait les passions coupables, à montrer le poignard qu'elles enfoncent dans le cœur de celui qui s'y laisse entraîner, et à nous révéler tous les affreux supplices auxquels elle le dévoue dès cette vie; constante à parler aux hommes un langage toujours conforme à celui que la vérité éternelle leur tient à tous dans le plus secret de leur conscience; savante à peindre le bonheur, les inexprimables délices que la vertu sait faire goûter au milieu même des plus rudes épreuves; abandonnant à l'ordre universel toutes les passions, qui sont la vie du cœur, elle les développerait en les épurant; elle identifierait avec la religion et par là même exalterait sans mesure tout ce qu'elles ont de légitime et de noble; et, comme les sentiments dont elle emplirait toutes les âmes ont eu dans tous les temps le privilège de transporter les hommes d'enthousiasme, à cause de l'affinité naturelle qu'ils ont avec la véritable gloire, ils ne pourront tarder à se manifester d'une manière publique et générale; cette manifestation puissant[e] réagirait à son tour sur les sentiments individuels qui l'auraient produite, pour les développer de plus en plus: et c'est ainsi que la littérature, fille et compagne inséparable de la religion, travaillerait de concert avec elle à l'œuvre divine dont elle est chargée, à la régénération complète et définitive du monde: semblable à elle, bien que différente par les formes qui lui sont propres, médiatrice entre elle et nos plaisirs qu'elle diviniserait par l'amour, elle serait vraiment sur la terre une image du Beau suprême, coéternelle splendeur de l'éternelle vérité qui l'engendre. ${ }^{37}$

Nous avons retenu ce long passage parce qu'il nous paraît représentatif des propos de Raymond dans les années à venir. L'essentiel de ses opinions s'y trouve. Les sources à tarir, les thèmes à développer et la façon de les traiter, les objectifs sociaux, voire religieux, que Raymond assigne à la littérature, tout ce fond ne connaîtra pas d'évolution. C'est en vain qu'on cherchera le pen-

37 Ibid., $11 \mathrm{~s}$. 
dant de la remise en question du credo conservateur sur le libéralisme et le gallicanisme faite par l'abbé en 1872, question qui précipita une levée de boucliers ultramontains ${ }^{38}$. En littérature, ce sera le calme plat. Ses opinions ultérieures corroboreront les prémisses posées dans les premiers textes, comme si la position de départ lui était à ce point vitale que, par après, tout commentaire est jugé superfétatoire. Certain de la valeur de son interprétation unitaire de la vie, - et le milieu aidant, - Raymond n'avait plus qu'à se cantonner dans la répétition.

On aura aussi remarqué le rôle social dévolu à la littérature dans les commentaires de Raymond. Il est clair que l'abbé accorde à l'imprimé une influence qu'il ne met point en doute. Il pense avec de Bonald que la littérature est l'expression de la société et il n'est pas loin de soutenir aussi «que la société est la réalisation de la littérature ${ }^{39}$ quand il écrit: «si la religion ne vient pas au secours de la littérature, celle-ci va bientôt mettre la société dans un état intellectuel et moral qui lui prépare et des ignominies et des désastres. ${ }^{40}$ »

Certes, pour saisir toute l'ampleur de cette interprétation de la réalité, il faudrait remettre cette citation dans le contexte historique qui l'a vu naître. Néanmoins, hors ce fait et tout en admettant qu'il y ait influence réciproque de la littérature et de la société, la position de Raymond dénote beaucoup de sévérité à l'égard de la littérature contemporaine, la française en l'occurrence. Il lui paraît normal de restreindre la liberté d'expression au point que le moraliste n'écrive que pour des moralistes. L'ordre social ainsi préservé, Raymond demande l'adhésion de l'écrivain à une unité de pensée et d'action, à une élévation spirituelle qui fondent toute la cohérence du monde de l'ultramontain. Mais, cette situation idéale, qui ne peut se

38 Le 8 décembre 1872, Raymond disait dans une conférence sur le culte à Marie qu'au Canada: «il n'y a pas de libéralisme dans le sens condamné par le vicaire du Christ; car il ne s'agit pas évidemment du libéralisme politique" et il ajoutait: "Ici point de gallicanisme» (Le Courrier de Saint-Hyacinthe, 14, 19 décembre 1872). Luc Désilets, un ultramontain verra dans ces propos «la pilule du diable recouverte de la fleur de la dévotion à Marie» [Luc Désilets à Mgr Laflèche, cité par Albert Tessier, "Luc Désilets, un des «fanaux de tôle» de Mgr Laflèche», Cahiers des Dix, 19 (1954): 176]. Le Nouveau-Monde, journal de l'évêché de Montréal, suggérait à Raymond de se défaire de l'influence des libéraux-catholiques français et du «lamennaisianisme reprouvé par Grégoire XVI». (Le Nouveau-Monde, 8 janvier 1872).

39 Nous ne pouvons manquer de faire une analogie avec les tenants de l'influence néfaste de la télévision sur les mœurs d'aujourd'hui.

40 J.-S. Raymond, «Nécessité de la religion dans l'éducation », RC (1874) : 606. 
retrouver que dans et par la religion, n'existait pas, surtout en France. Dans la même logique, il élève le métier d'écrivain au rang de "sacerdoce» ${ }^{41}$, - ou de héros romantique - en lui conférant des responsabilités rattachées à cet état, dont celui d'enseigner le bien pour le salut des âmes ${ }^{42}$. Toute création devient une médiation entre l'homme et le surnaturel. Inspiré par la religion, l'œuvre sert la société. D'où la nécessité d'une conscience omniprésente et d'un enseignement, puisque "la société en s'imprégnant de l'esprit des écrits qui sont lus partout avec anxiété met bientôt en pratique les principes et les sentiments qu'elle puise de toutes parts, et qu'elle se modèle sur les types que l'art lui a présentés» ${ }^{43}$. On comprend mieux alors l'opposition de principes de Raymond à la théorie de l'art pour l'art, car «une société qui subirait sans protestation cette maxime se livrerait bientôt aux vices et aux passions». L'art ne peut être une fin en soi sans but moral et social, puisque «la religion seule est la maîtresse de l'esthétique, c'est-à-dire de la science qui détermine le caractère du beau dans les productions de la nature ou de l'art ${ }^{44}$.

Cette idée n'est pas nouvelle. Ce qui n'a pas encore été formulé toutefois, et qui s'insère aisément dans la théorie antérieure du beau moral, Raymond le dira dans un texte publié en 1874:

La forme n'est pas indifférente au point de vue moral [...] un écrivain peut employer certaines formes piquantes qu'agrée la légèreté des esprits et la mollesse des coeurs; elles excitent une admiration qu'une raison droite et élevée devrait leur refuser; elles produisent une satisfaction plutôt sensuelle qu'intellectuelle,

41 Ibid., 602.

42 Aux étudiants qui compteraient faire du journalisme il dira: «...ne perdez pas de vue la grande responsabilité que vous assumez. Songez à l'effet que vos paroles ou votre plume aura nécessairement sur un certain nombre de vos compatriotes; un bien ou un mal d'une portée peut-être considérable est entre vos mains. [...] Rendez-vous aptes [...], et surtout suppléez à ce que l'âge vous refuse de sagesse et d'expérience en consultant les hommes dont le jugement et l'amour pour le bien vous sont connus; et faites-vous un devoir de déférer à leurs avis.» [J.-S. Raymond, «Discours sur l'importance des études classiques», Foyer Canadien (1866): 156]. Ou encore: "Tout homme qui écrit doit se demander quel sera l'effet de son livre sur les âmes. Produira-t-elle en elles une impression vers ce qui est grand, ce qui est bien, ce qui est bon...» (Raymond, Nécessité... op. cit., 602).

43 J.-S. Raymond. De l'intervention du prêtre dans l'ordre intellectuel et social, $2^{\mathrm{e}}$ éd. (Saint-Hyacinthe, les Presses à pouvoir du "Courrier»1877), 25. Raymond présente cette idée sous forme interrogative. Ce n'est point là une hypothèse réelle mais un procédé de style.

44 Raymond, Nécessité..., op. cit., 603. 
qui nuit à la rectitude et à la force de l'esprit: elles pervertissent le goût. ${ }^{45}$

L'expression se voit circonscrite: l'orthodoxie religieuse régit le fond et la forme. Certes nous voyons mal aujourd'hui comment Raymond pouvait juger du «langage sans règle, sans dignité, désordonné [...] abject » ${ }^{46}$ d'un auteur. L'entreprise nous paraît pour le moins hasardeuse. Mais, en acceptant que manière et contenu s'appuient et se soutiennent, et surtout qu'il existe une forme idéale et universelle de bien dire à laquelle correspond un bien penser moral, on peut suivre la pensée de Raymond. Nous croyons que ses arguments contre l'expression sont un pendant de seconde zone à l'appui de ceux qui visent le fond ${ }^{47}$.

Il ne faudrait pas croire que seule la littérature subit les rigueurs de Raymond. Ses vues embrassent tous les champs de l'activité intellectuelle, lesquels, on s'en souvient, font partie de la définition de la littérature au XIX ${ }^{\mathrm{e}}$ siècle. Nous pourrions multiplier les exemples qui en font foi. Entre autres, l'histoire «un des plus importants objets offert à l'étude de l'homme» ${ }^{48}$ doit être au service de «la première de toutes les sciences, la religion ${ }^{49}$; la philosophie «devient une arme puissante à l'aide de laquelle le chrétien peut défendre la doctrine religieuse qu'il professe ${ }^{50}$; et quand Raymond se défend «d'être ennemi de mon [son] siècle» et contre l'industrie, il réplique:

Honneur donc et encouragement à l'industrie, pourvu toutefois qu'elle soit contenue dans de justes bornes, et que ses progrès

45 Ibid., 602. Dans une page rayée du manuscrit de cet article, Raymond écrit que la religion fait éviter «les excentricités d'idées et de langage». ([Raymond], «La religion et la littérature», ASSH, FR, A5, R3-env. 13, f. 25.) Raymond oubliait la «satisfaction plutôt sensuelle» des textes de plus d'un mystique... et des siennes (voir n. 79).

46 Ibid., 606.

47 Sur la question du gaumisme, Raymond oppose l'enseignement bénéfique des Pères de l'Église à l'influence délétère des Anciens; le reproche qu'il adresse aux classiques, c'est de n'être pas assez chrétiens, - sans parler des auteurs du XVIII e siècle.

48 Raymond, «Discours sur l'importance...», op. cit., 111.

49 Raymond, "Étude sur le Moyen Âge», RC (1867): 753. Le Troisième discours de la Nécessité de la religion dans l'éducation (RC (1874): [595]-600) porte sur la religion et l'histoire. Raymond y défend la thèse que la religion et l'histoire ne saurait pas se passer l'une de l'autre. L'étude de l'histoire peut aider à prouver et à faire voir les bienfaits du christianisme. Au service de la religion, elle devient une arme de défense.

50 Raymond, «Entretien sur les études classiques», RC (1872): 676. 
ne fassent pas dominer les intérêts matériels sur l'esprit religieux et moral, dans lequel consiste essentiellement la véritable civilisation, et la prospérité réelle des nations. ${ }^{51}$

En fait, on le devine depuis le premier exemple, «...la religion est appelée à décider toutes les questions non seulement de l'ordre surnaturel, mais de l'ordre social, philosophique, littéraire même»" ${ }^{52}$. Pour Raymond «toutes les sphères où s'exercent l'intelligence humaine relèvent donc de la religion. Chacune d'elles doit rendre hommage au Seigneur qui est le Dieu des sciences. ${ }^{53}{ }$ Ces positions ressortent d'une conception religieuse de la vie.

L'engouement de l'abbé mascoutain pour le moyen âge ${ }^{54}$ s'éclaire du même coup. Il s'explique certes par le goût mis à la mode par les romantiques et les traces laissées par la lecture de Chateaubriand, mais surtout, croyons-nous, par l'admiration suscitée par cet âge de foi où la place faite à la spiritualité répond au monde idéalisé de Raymond. À cet enthousiasme correspond une contrepartie à l'égard de «cet événement si mal appelé la Renaissance des lettres, qui amena le paganisme partout ${ }^{55}$. Mais les regrets de l'abbé sur l'imitation des Anciens ne l'empêcheront toutefois pas de recommander chaudement les classiques. "Lisez les grands maîtres, répétaitil, relisez-les, et je dirais presque ne lisez qu'eux. ${ }^{56}$ " École de goût, de grandeur, de noblesse, d'autorité, le classicisme trouvait chez lui un accueil favorable puisque "l'inspiration chrétienne, reprendra-t-il après Chateaubriand, est le principe des plus grandes beautés des chefs-d'œuvre de cette époque. ${ }^{57}$ » Au XVIII ${ }^{e}$ siècle littéraire français, l'abbé est tenté de lui attribuer un mot de de Maistre sur Voltaire: «Dieu ne l'aime pas. ${ }^{58}$ " Faut-il ajouter: Raymond non plus?

C'est aussi en fonction de sa concordance avec la religion que se manifeste l'intérêt de Raymond pour la littérature du XIX S'il fait la recommandation de lire les grands écrivains de l'époque, «ceux surtout dont la religion a si éloquemment inspiré le génie» 59 ,

51 Raymond, «Étude sur le Moyen Âge », op. cit., 921.

52 Raymond, "Destinée providentielle de Rome», RC (1864): 763. op. cit., 12.

53 Raymond, De l'intervention du prêtre dans l'ordre intellectuel et social,

54 Raymond, «Étude sur le Moyen Âge», RC (1867) et (1868).

55 Ibid., 837.

56 Raymond, «Discours sur l'importance... », op. cit., 118.

57 Raymond, «Nécessité...», op. cit., 605.

58 Raymond, «Discours sur l'importance...», op. cit., 118.

59 Ibid. 
c'est pour mieux décrier «cette multitude de romans et de drames» et multiplier ses avertissements contre «cette littérature sans principe et sans règle ${ }^{60}$, "cette littérature sans frein [...] qui consacre tous les caprices de l'écrivain, pervertit le goût, et finit par éteindre le génie par l'excès de la licence où elle l'abandonne ${ }^{61}$. On aura compris que la majorité des grands noms de la littérature du $\mathrm{XIX}^{\mathrm{e}}$ fait partie de la seconde catégorie, surtout les romanciers.

Comme plusieurs de ses contemporains ${ }^{62}$, Raymond ne prise guère le roman. Et c'est au nom de la vocation tout utilitaire de littérature qu'il condamne ce genre:

De prétendues esquisses de mœurs, des aventures romanesques hors de toute vraisemblance, des futilités de tout genre, où souvent la bizarrerie de la forme le dispute à la mise du fond. Là rien qui instruise, qui excite un sentiment généreux, qui élève le caractère: là souvent, sous des déguisements plus ou moins habiles, les passions se montrent avec l'intention de séduire. ${ }^{63}$

Il est réfractaire au genre romanesque au point de ne pas recommander la lecture des romans historiques, lesquels s'approprient la vérité pour la déformer au gré de la fiction ${ }^{64}$. Il doute même des résultats obtenus par ceux qui l'utilisent pour des fins morales ${ }^{65}$. Dans cette catégorie d'œuvres, il fait toutefois deux exceptions: Fabiola du Cardinal Wiseman et les Fiancés d'Allessandro Manzoni, romans qualifiés de chefs-d'œuvre, «où la morale est la plus pure » ${ }^{66}$.

Au chapitre des proscriptions, il pousse ses convictions jusqu'à souhaiter aux étudiants de son collège d'avouer, à la fin de leur vie, comme Denis-Benjamin Viger, de n'avoir jamais lu de roman. Et à ceux qui s'y adonnent, il prédit l'affadissement du cœur et le manque de $«$ force virile» ${ }^{67}$.

Si le roman est traité comme le dernier des genres, la poésie, «la plus noble expression des plus nobles pensées de l'être intelligent,

\footnotetext{
$60 \quad$ Ibid., 119.

61 Raymond, «Entretien sur l'éloquence...», op. cit., 20.

62 Cf. Dostaler, Les Infortunes..., 61-109.

63 Raymond, «Entretien sur les études...», op. cit., 614.

64 Raymond, «Discours sur l'importance...», op. cit., 152.

65 Ibid.

66 Ibid., 153.

67 Raymond, «Entretien sur les études...», op. cit., 674.
} 
dira-t-il à la suite de Bonald ${ }^{63}$, est reçue avec plus de discernement. Même si «elle a été donnée à l'homme pour célébrer dans le langage le plus sublime Dieu et ses œuvres» ${ }^{69}$, Raymond rappelle, position presque platonicienne, que les lecteurs, qui «n'aiment que l'utile et le vrai», pourront se passer de cet «amusement frivole», les autres pourront toujours accorder «un sourire passager à ses frivoles attraits ${ }^{70}$.

Du théâtre, autre que classique, il n'en est pas question dans les écrits de Raymond ${ }^{71}$, sinon pour exprimer des réserves à l'égard de Molière, «dont l'immoralité, en certaines productions, n'a point été dépassée par celle des dramaturges de nos jours ${ }^{72}$.

C'est au nom de l'utilité des textes dans la formation morale, de la vérité à connaître et à défendre, des appels à l'intelligence plus qu'à l'imagination ou à la sensibilité que l'abbé marque sa préférence pour les écrits que nous identifions aujourd'hui comme essai. Constamment tourné vers l'utile et le sérieux, Raymond, - que des jeunes qualifieront «d'exigeant, [d'] étroit, [d'] austère ${ }^{73}$ et de «grave» ${ }^{74}-$, ne fait guère de place à la fiction. Il la garde pour suspect. Nous dirions qu'il pense de la littérature comme de la presse: c'est un mal nécessaire. Et c'est parce qu'elle «véhicule des renseignements de la plus grande utilité pour la cause de la vérité 75 , qu'il l'accepte, avec réserve. On comprend son penchant pour les hagio-

68 Ibid., 673.

69 Ibid.

70 Raymond, «Discours sur l'importance...», op. cit., 114.

71 Raymond a préparé pour les cérémonies officielles du collège quelques pièces, tragédies ou drames inspirés d'œuvres connues, qui furent jouées par les élèves de la maison ou par ceux du couvent des Sœurs de la Présentation de Saint-Hyacinthe. On y trouve: "Balthazar, Daniel et les jeunes juifs captifs», "Catalina ou Rome sauvé», "Oreste», «Jonathas», "Fabiola», "L'école militaire», "Achille», "Le Martyre de Sainte-Catherine». L'une d'elles, préparée en huit jours de travail, ayant L.-A. Dessaules dans le rôle de Louis XIV, intitulée, "Procès du chef des Iroquois envoyé captif en France par M. de Rouville", est de l'invention de l'abbé, sauf pour une partie empruntée au Natchez de Chateaubriand (ASSH, Archives 1, 45; ASSH, FR A5 R2, b. 15-24, dossier 18).

72 Raymond, "Discours sur l'importance...», op. cit., 117.

73 Henriette Dessaulles (Saint-Jacques), Journal d'Henriette Dessaulles (Montréal, Hurtubise HMH, 1971), 224.

74 Oscar Dunn à François Tétreau, 3 janvier 1865, ASSH, Fonds Tétreau.

75 Raymond, «Discours sur l'importance...», op. cit., 154. 
graphies et qu'il écrive: «l'apologie chrétienne est la plus belle des littératures ${ }^{76}$.

À examiner les auteurs que Raymond se plaît à citer ou à commenter dans ses écrits ${ }^{77}$, on constate qu'il met en pratique ses propres enseignements. Celui dont le nom revient constamment, «la plus belle intelligence littéraire de ce siècle ${ }^{78}$, c'est Chateaubriand, qu'il pastiche à l'occasion ${ }^{79}$ et chez qui l'abbé mascoutain semble avoir retenu quantité d'idées ${ }^{80}$ et surtout la méthode: démonstration en faveur du catholicisme, mise en valeur de la révélation en faisant appel à l'exaltation ${ }^{81}$. Viennent ensuite de Maistre et les disciples de

76 Ibid., 147. À propos de l'histoire de Notre-Dame de Lourdes de Henri Lasserre, il écrit: "Il n'est aucune épopée, aucun drame, aucun roman qui offre un si saisissant intérêt que la lecture de ce livre où se révèle d'ailleurs le talent d'un écrivain supérieur." [Raymond, Discours sur l'action de Marie dans la société, prononcé devant l'Union catholique de St. Hyacinthe, le 8 décembre 1872. Nouv. éd. rev. et augm. (Québec, Ovide Fréchette, 1873), 58.]

77 Conscient des limites d'une telle méthode, qui n'a rien d'une analyse d'influence, nous nous en servons comme indicatif.

78 Raymond, "De l'Église et de l'État», RC (1866): 56. Il disait deux années plus tôt à propos de Mgr Gerbet: «depuis quelques années, il pouvait être regardé comme le plus grand écrivain alors existant». (Raymond, Destinée..., op. cit., 535.)

79 À titre d'exemple: «Bien souvent j'avais erré sous les arceaux de ses (du Colysée) vastes portiques; je m'étais assis au milieu de son arène; j'avais visité le monument dans toutes ses parties; c'était ma promenade favorite; j'y passais des heures entières. Mes sens et mon âme trouvaient dans la vue du Colysée et dans les souvenirs qu'il rappelle une jouissance plein de charme et de grandeur. Dans les jours, et ils sont fréquents à Rome, où la ville des Césars et des Papes, m'avait donné dans quelques-unes de ses ruines fameuses ou de ses sanctuaires pleins de grâces, quelque forte émotion, qui exaltait toutes les puissances de mon âme, $j$ 'aimais sur le soir à venir y associer l'impression de l'aspect et des réminiscences du Colysée. La corde de la lyre du cœur que ce lieu touchait complétait la délectable harmonie des sentiments qui vibraient au-dedans de moi. Le Ciel secondant les inclinations de mon âme m'avait réservé ma plus magnifique jouissance au Colysée, pour le jour où j'avais éprouvé d'ailleurs les plus touchantes émotions que Rome peut présenter à un cœur chrétien. (Ibid., 540).

80 Entre autres que de toutes les religions, la chrétienne est la plus favorable aux lettres, aux arts, aux sciences, à la société et à la liberté; que la civilisation chrétienne est supérieure aux autres et que l'irréligion crée une littérature inférieure.

81 Par exemple, tout l'article "Étude sur le moyen âge» tente de démontrer la vertu sociale du christianisme; et cet autre, intitulé «Destinée providentielle de Rome» veut appuyer le pouvoir temporel du Pape par le biais de la grandeur du christianisme, les beautés de la ville éternelle, historique et physique: «Rome convient à l'Église et l'Église convient à Rome. Rome est grande et belle, nous croyons l'avoir prouvé, et sa grandeur et sa beauté sont dues à ce que la religion a fait pour elles. Otez-en, en effet, la religion de Rome et ce caractère sacré, mystérieux, poétique, que nous lui avons reconnu, ne peut s'expliquer. C'est évidemment l'Église qui a fait 
Lamennais, sur lesquels Raymond semble avoir jeté son dévolu après la défection du maître, puis les grands noms du catholicisme au XIX ${ }^{\mathrm{e}}$ siècle, les orateurs, les hommes politiques, les historiens et à l'occasion les critiques et les littéraires ${ }^{82}$.

Soulignons, tout en laissant au biographe de Raymond le soin d'élucider la question, que l'abbé ne semble jamais faire grand cas de l'idole des ultramontains, Louis Veuillot, écrivain dont la filiation spirituelle avec les mennaisiens est certaine et dont l'influence sera plus que séculaire au Canada ${ }^{83}$. Il y va de même pour Lamartine, poète qui jouit d'une grande ferveur parmi les contemporains de Raymond. Dans ses écrits, l'abbé reste muet à son sujet. Rangeait-il l'auteur des Méditations parmi ceux dont la lecture «n'est plus qu'une sensation ${ }^{84}$ ? Avait-il perçu la «fade religiosité» du poète? Il faut

\footnotetext{
Rome ce qu'elle est, et qui donne même, j'ose le dire, à son passé la grandeur dont il jouit parce qu'elle unit d'une manière admirable et dans leur histoire et dans leurs monuments Rome ancienne et Rome moderne en une destinée commune où l'une complète l'autre. Si l'Église a fait Rome belle comme elle l'est, qu'on lui laisse le soin de conserver sa beauté, mais pour que l'Église agisse sur Rome matérielle, il faut qu'elle soit maîtresse à son égard; cela est de toute évidence. [Raymond, "Destinée providentielle de Rome», RC (1864): 755].

82 Par ordre décroissant de rappel: Montalembert, Lacordaire, Gerbet, Guizot, de Bonald, Bossuet, Voltaire, Rousseau, Thiers, Hugo, Mme de Staël, Fénelon, Thierry, Lamennais, Rohrbaker, Cortès, Veuillot, Massillon, Corneille, Racine, Dante, Ozanam, Descarte, Montesquieu, La Harpe, Dupanloup, Bourdaloue, Wiseman, Sainte-Beuve, Michelet, Rochefort, Renan, Froissart, Erasme, Molière, Goethe, Byron, Dumas, Russell, Davy, Brougham, Hauster.

83 L'abbé Raymond avait pourtant l'habitude de noter, au cours des années, les articles importants du journal de Veuillot, l'Univers. Il procédait de la même façon avec le Monde et l'Université catholique (ASSH, FR, R2, sec. A; b. 15-24, dossier 24.) Il se cachait sûrement quelques vérités dans les propos que tenait l'abbé Alphonse Villeneuve dans un pamphlet contre Raymond en 1872. Villeneuve écrivait: "À différentes personnes et à mille reprises, monsieur Raymond s'est déclaré l'adversaire de Louis Veuillot, de l'Univers, de Mgr de Tulle, disant du premier qu'il fait à la religion un mal incalculable, qu'il compromet tout et qu'il bouleverse tout dans l'Église». [Binan, pseud. Alphonse Villeneuve. Le Grand Vicaire Raymond et le libéralisme catholique (Montréal, Le Franc-Parleur, 1872), 8.] Raymond avait-il suivi Montalembert ou Lacordaire à propos de l'Univers et de son rédacteur? (Robert Sylvain, La Vie et l'euvre de Henri de Courcy, 1820-1861. Premier historien de l'Église aux États-Unis (Québec P.U.L., 1955), 282; Quelques aspects..., op. cit., 278. 84 Raymond, Entretien sur les études..., op. cit., 603.
} 
le croire ${ }^{85}$. Bien renseigné sur ce qui se disait en Europe, son enthousiasme, s'il en eut, dut s'attiédir dès 1835 ou $1838^{86}$.

Raymond n'a pas porté de jugement sur les auteurs du Québec. Il s'est limité à des commentaires généraux et à signaler, surtout au conditionnel, les dangers individuels et sociaux de la diffusion grandissante du roman français au pays. Il suggérait aussi aux écrivains d'ici de s'engager dans d'autres voies que celle du roman ${ }^{87}$. Somme toute, en 1874, satisfait des « œuvres d'apologétique chrétienne, d'histoire, d'éloquence, de grande et noble poésie» qui constituent la littérature naissante de la patrie, l'abbé Raymond l'incite à puiser aux mêmes sources ${ }^{88}$ et à maintenir son lien avec la religion. "Une saine et brillante littérature, dira-t-il, serait un des éléments les plus puissants de notre nationalité. ${ }^{89}$ "

L'univers littéraire de l'abbé Raymond repose sur une grille d'interprétation tirée des idées mennaisiennes et dont la théorie du sens commun est la pierre d'angle. Fort de cette dialectique du beau moral, qui jette un pont entre la vérité catholique et la beauté littéraire; appuyé sur les mots d'ordre et la vision chrétienne des de Bonald, de Maistre et surtout de Chateaubriand, puis, plus tard, sur celle des disciples des Lamennais, Raymond propose de la littérature une vision unifiée par la foi dans ses données, hermétique et immuable parce que globalisante.

85 Le seul indice que nous ayons des opinions de Raymond sur le poète se trouve sur quelques feuillets rédigés à l'occasion des lectures de l'abbé. Raymond tire quelques notes à propos du Cours familiers de littérature (1856-1869). On y voit des commentaires du genre: «L'amour de la forme, voilà tout Lamartine»; "négation de la création", et sur le 5 e entretien: "c'est la glorification de l'amour sensuel. Je ne conçois pas comment les jeunes filles [à qui] le christianisme a fait le don de maternité, puisse[nt] lire cet entretien». (ASSH, A5-b5, 62-85, dossier 80).

${ }^{86}$ Cf. Robert Sylvain, «Lamartine et les catholiques de France et du Canada» RHAF (1950): [29]-60; [233]-248; [375]-397.

${ }_{87}$ Raymond, Nécessité de la religion..., op. cit., 606.

88 Les propos de Raymond vont dans le même sens que ceux de l'abbé HenriRaymond Casgrain: «La beauté et la grandeur des dogmes catholiques source inépuisable de profondes contemplations et de sublimes inspirations; les principes de l'ordre social et moral à défendre contre tant d'attaques dont ils sont l'objet; les faits héroïques dont notre histoire nationale abonde; ces belles mœurs canadiennes qui faisaient naguères, et qui, jusqu'à un certain point encore aujourd'hui, font l'honneur de notre patrie: cette terre du Canada, si magnifique par les fleuves qui l'arosent, et par la beauté et la variété de ses sites tour-à-tour si gracieux si pittoresque, si grandiose: voilà une noble matière offerte à ceux à qui le ciel accorde le don du génie.» (Ibid., 607).

89 J.-S. Raymond, Discours sur la nécessité de la force morale (Montréal, Plinguet et Laplante, 1865), 45 . 
La méthode en elle-même présente certains dangers. Faire du surnaturel l'élément essentiel de toute production de l'esprit ouvre la porte à une interprétation uniquement morale de la littérature; se limiter au consentement universel pour jauger les œuvres littéraires, c'est, à priori, verser dans le conservatisme, du moins l'attentisme inhérent à cette approche. Car, il est impossible de s'appuyer sur une tradition de jugement pour évaluer les œuvres contemporaines.

En fait, l'abbé subordonne la littérature à la religion: soutenir et servir deviennent un leitmotiv. Il lui impose des objectifs moraux et sociaux, lui suggère des thèmes nobles et une manière noble de dire. L'esthétique est reléguée au second plan puisque la littérature est avant tout école d'édification, de vertu et de formation aux bonnes mours. À ses yeux, la fiction a des airs de liberté plus que suspects: ses proscriptions à l'égard de toute forme de roman en font foi. Seuls l'utile, le beau moral, les auteurs dont l'orthodoxie ne peut être mise en doute, les périodes de l'histoire et les écoles littéraires à forte inspiration chrétienne sont valables. Placé dans cette vision particulière, à ses yeux le Québec littéraire de l'époque faisait figure d'avant-garde.

Nous voyons mal toutefois comment Joseph-Sabin Raymond, tributaire des idées qui ont cours à son époque, engagé dans le renouveau religieux de son temps, les luttes de pouvoir entre le spirituel et le temporel, la question romaine, l'Église et l'État, le laï et le clérical, comment ce prêtre, ayant charge d'âmes et hanté par des préoccupations d'ordre religieux, eût pu faire un traitement de faveur à la littérature. Sa vision ultramontaine surtout, qui s'insère dans une conception et un mode de vie plus vastes, n'est pas unique. En effet, nombreux sont les laïcs qui, en même temps que lui ou à sa suite, sans avoir jeté les mêmes prémisses et vécu des mêmes préoccupations, s'approprieront les conclusions que commandent cette façon de voir.

Suivis à la lettre et conduits à leur aboutissement logique, les certitudes, les conseils, la méthode de l'abbé menaient droit à la «théocratisation» de la littérature. Ils forment, chose certaine, un des chaînons d'un concensus d'opinions, ou de ce que l'on a qualifié de barrage de la critique ${ }^{90}$, qui, au milieu du XIX ${ }^{\mathrm{e}}$ siècle, s'est opposé au développement d'une littérature d'imagination au Québec.

90 Léopold Lamontagne, «Les Courants idéologiques dans la littérature canadienne-française du XIX siècle», RS, V, 1-2 (1964): 119. 